\title{
Does confined water exhibit a fragile-to-strong transition?
}

\author{
J. Hedström ${ }^{1}$, J. Swenson ${ }^{1}$, R. Bergman ${ }^{1}$, H. Jansson ${ }^{1}$, and S. Kittaka ${ }^{2}$ \\ 1 Department of Applied Physics, Chalmers University of Technology, 41296 Göteborg, Sweden \\ e-mail: johan.hedstrom@chalmers.se \\ 2 Department of Chemistry, Faculty of Science, Okayama University of Science, 1-1 Ridaicho, \\ Okayama 700-0005, Japan
}

\begin{abstract}
The dynamics of supercooled confined water has recently been shown to have a pronounced, apparent fragile-to-strong transition (FST). Here we use broadband dielectric spectroscopy $\left(10^{-2}-10^{9} \mathrm{~Hz}\right)$ to study the dynamics of water confined in silica matrices MCM-41 C10 and C18, with pore diameter of 21.4 and $36.1 \AA$, respectively. The local dynamics of water molecules and the dynamics of the hydroxyl groups on the inner wall of the pores are followed up to over $240 \mathrm{~K}$. We argue that the reported FST for confined water is due to the vanishing of the cooperative $\alpha$ relaxation, which implies that it should not be interpreted as a true FST.
\end{abstract}

\section{Introduction}

There are several tantalising anomalies of water, such as the famous density maximum at $277 \mathrm{~K}$ and the power law divergence at $228 \mathrm{~K}$ [1]. In particular, dynamics related to the glass transition has been subject to many experiments on both bulk and confined supercooled water. The latter is important since confined water with a reduced mobility is of key importance for the functions of biological molecules. Confining water is also a way to avoid crystallisation at $\mathrm{T}=150 \mathrm{~K}$ to the homogeneous nucleation temperature $(\mathrm{T}=231 \mathrm{~K})$.

The glass transition temperature $\left(T_{g}\right)$ of water has been widely debated. The long accepted value of $136 \mathrm{~K}[2,3]$ has been questioned [4-7] and proposed rather to be around $165 \mathrm{~K}$ for bulk water. Water is extraordinarily fragile (non-Arrhenius temperature dependence of the viscosity) close to the melting point and does not extrapolate to the suggested $\mathrm{T}_{g}$. A possible fragile-to-strong transition (FST) was suggested [8] to resolve this issue and since then several experiments [9-12] indicate the existence of such FST.

The need for a transition was noticed in the study of water confined in hydrated Na-vermiculite clay $[9,13,14]$ where the confinement is in one dimension only. The extrapolation of high temperature data from quaielastic neutron scattering (QENS) did not agree with the low temperature dielectric data. However an actual transition was not seen. Later, a transition was detected in the dielectric spectrum of water confined in molecular sieves of pore size $10 \AA[10,15]$. These changes in the temperature dependence were however not explained by a true FST, but by the merging of a nonobservable $\alpha$ relaxation with a local $\beta$ relaxation.

A pronounced apparent FST was seen from QENS data [11] of water confined in nanoporous silica (MCM-41) with pore sizes 14 and $18 \AA$ at 222 and $225 \mathrm{~K}$ respectively. The phenomenon was explained as a transition from a high density liquid to a low density liquid [16]. Recently a FST at $215 \mathrm{~K}$ was reported [12] in a QENS measurement of translational motions of surface water in a quasi-two-dimensional confinement of cerium oxide $\left(\mathrm{CeO}_{2}\right)$. Previous QENS studies of confined water in MCM-41 [17] have not reached such low temperatures. 
The QENS data $[11,12]$ describes a VFT-dependence at high temperatures and an Arrhenius dependence after the apparent transition, with an activation energy several times smaller than the corresponding activation energy found at lower temperatures for water in MCM-41, molecular sieves and a wide range of different confining matrices [4]. In this work we try to overbridge this discrepancy by investigating the dynamics of water confined in MCM-41 with pore diameter 21.4 and $36.1 \AA$. The broad frequency range, $10^{-2}-10^{9} \mathrm{~Hz}$ (eleven orders of magnitude), makes it possible to follow the main relaxation of water to temperatures about $240 \mathrm{~K}$ and investigating the possible existence of a FST.

\section{Sample preparation and experiment}

Nanoporous silica of type MCM-41 was prepared by the modified Beck method [18]. Long-chain alkyltrimethylammonium bromides, $\mathrm{C}_{n} \mathrm{H}_{2 n+1}-\left(\mathrm{CH}_{3}\right)_{3} \mathrm{~N}^{+} \mathrm{Br}$, were used as the template organic reagent. The pore diameter is set by the number of carbon atoms, $n$, in the long alkyl group. The samples used in this work had $n=10$ and 18, subsequently called C10 (21.4 $\AA$ ) and C18 (36.1 $)$. The inner walls of the samples are covered with hydroxyl groups. The samples were hydrated by exposing them to $100 \%$ humidity at room temperature after evacuating at $415 \mathrm{~K}$ for four days. At this temperature the water disappeared from the sample but not the hydroxyl groups. The samples not exposed to humidity but only evacuated at $415 \mathrm{~K}$ are labelled "dry". Note that the size of the pores allows not more than three layers of water molecules and OH-groups from the inner walls to the centre of the pores for the C10 sample and a maximum of five layers in the C18 sample. Previous DSC measurements on these samples show that the water in the C10 sample (21.4 $\AA$ ) does not crystallise [17]. The broadband dielectric measurements were performed using two different analysers for the frequency range $10^{-2}-3 \cdot 10^{6} \mathrm{~Hz}$ (Novocontrol, Alpha) and $10^{6}-10^{9} \mathrm{~Hz}$ (HP, 4192). Electrodes of 20 and $10 \mathrm{~mm}$ radius for the broadband and high frequency analyser respectively were used.

\section{Results and discussion}

In Fig. 1(a) the imaginary part of the dielectric constant $\left(\epsilon^{\prime \prime}\right)$ for the C10 sample is shown for different temperatures. For $T>170 \mathrm{~K}$ a prominent low frequency power-law contribution, due to polarisation effects, enters the spectrum. This is noticed in previous studies of MCM-41 [19] and dominates the data for low frequencies and high temperatures.

The spectrum is curve-fitted using two symmetric Cole-Cole functions, $\epsilon=\epsilon^{\prime}(\omega)-i \epsilon^{\prime \prime}(\omega)=$ $\epsilon_{\infty}+\sum_{i=1}^{2} \Delta \epsilon_{i} /\left[1+\left(i \omega \tau_{i}\right)^{\alpha_{i}}\right]$. A detailed discussion of the analysis and fitting procedure of the spectra will be given elsewhere [20]. For the dry sample a single loss peak dominates the spectra. When the hydration level is increased the relative dielectric strength of this process decreases and a new, slower process enters the spectrum (see Fig. 1(b)). The strength of the slow process increases with increasing hydration level until it settles (at a level more than one order of magnitude larger than the fast process) when the pores are about half-filled. In Fig. 1(b) the average relaxation times $\left(\tau_{i}\right.$ of the Cole-Cole function) of the two processes are shown for different hydration levels. The fastest process is attributed to the hydroxyl groups at the pore walls. The temperature dependence of the relaxation time is in agreement with the dynamics of the hydroxyl group found in dry MCM-41 with pore size $32 \AA$ [19]. The slower process is not visible in the dry sample and therefore associated with the supercooled water in the pores. The activation energy $\left(E_{a}\right)$ of the water process is similar to $E_{a}$ of the main relaxation found for water confined in a large number of systems [4]. This process has recently been attributed to a local process and is therefore not directly related to the dynamic glass transition. This work supports this conclusion by the absence of a calorimetric glass transition, the hydration independent activation energy and the symmetric shape of the relaxation peak.

In Fig. 1(c) the dielectric data are compared with the QENS data [11,16]. It should here be noted that normally there exist an empirical equivalence between relaxation times from dielectric data and from QENS at $Q=1 \AA^{-1}$ [21], which is a typical $Q$-value for a QENS 


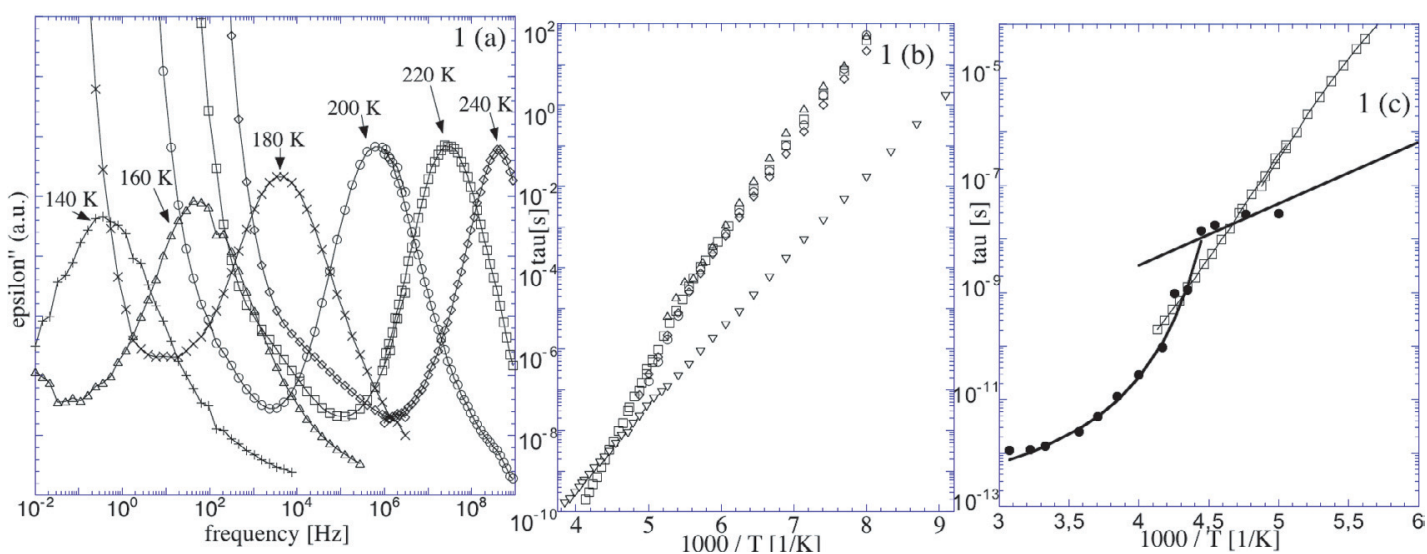

Fig. 1. Dielectric loss $\left(\epsilon^{\prime \prime}\right)$ at different temperatures for MCM-41 C10 (21.4 $\left.\mathrm{A}\right)$ with hydration level $\mathrm{H}=22 \mathrm{wt} \%$ for the frequency range $10^{-2}-10^{6} \mathrm{~Hz}$ and $\mathrm{H}=26 \mathrm{wt} \%$ for $10^{6}-10^{9} \mathrm{~Hz}$. (b) Arrhenius plot for the process from hydroxyl groups $(\nabla)$ of the dry sample and for the water process in MCM-41 C10 with $\mathrm{H}=12 \mathrm{wt} \%(\diamond), 22 \mathrm{wt} \%(\square), 55 \mathrm{wt} \%(\mathrm{O})$ and in MCM-41 C18 (pore diameter 36.4 A) with $\mathrm{H}=39 \mathrm{wt} \%(\triangle)$. Note that the relaxation time from the water process is virtually unaffected by the hydration level and the two different confinement sizes. (c) Arrhenius plot of MCM- $41 \mathrm{C} 10, \mathrm{H}=55 \mathrm{wt} \%$ from dielectric spectroscopy $(\square)$ and fully hydrated MCM-41 (pore size $18 \AA$ ) by QENS (-) [11].

experiment. In Fig. 1(c) a large discrepancy in the temperature dependence of the relaxation time as measured by the two techniques. What are the possible explanations for this dual picture? The effect on the dielectric processes was very small when reducing the confining pore diameter from 36.1 to $21.4 \AA$ (or even down to $10 \AA$ in the case of molecular sieves [10]) and the QENS data were not substantially altered when substituting the $18 \AA$ pores for the $14 \AA$ ones. This makes it unlikely that the smaller confinement of the QENS data should cause this discrepancy. We can also rule out differences in the cooling or heating of the sample since dielectric data are reproduced when collecting data on cooling.

Reasonable explanations to the discrepancy could either be the limited resolution of the QENS measurements at the lowest temperatures or that the two experiments are not probing the same dynamics. The dielectric loss peaks originate from reorientations of dipole moments and the largest ones in these samples are, by far, those from water molecules and hydroxyl groups. The neutrons however, probe essentially the protons present in the sample since the incoherent cross section for hydrogen is much larger than for any other isotope present in the samples. Therefore, the neutrons are sensitive to motions of both water molecules and single protons. A decoupling of the translational dynamics of protons from the dynamics of the water molecules is possible when the water becomes less mobile. The neutrons can therefore probe a mixture of average relaxation times for translational proton diffusion and reorientational motions of water molecules. Another possible explanation is that the QENS experiment is probing a very local process not present, or very weak, in the dielectric data. This process should exhibit an even more local behaviour than the ordinary $\beta$-like process.

The dielectric data extrapolates to unrealistic relaxation times $\left(\tau \sim 10^{-25} \mathrm{~s}\right)$ at infinite temperatures. It is therefore probable that the Arrhenius process would follow the neutron data at higher temperatures. A change in the temperature dependence of the relaxation time is therefore expected somewhere in the measured temperature range. A kink is also seen in the dielectric data at $\sim 180 \mathrm{~K}$. Williams' product ansatz [22] explains the apparent change in the activation energy of the local $\beta$ process at temperatures where it merges with the $\alpha$ process [23]. The absence of a calorimetric glass transition [17] indicates that the cooperative $\alpha$ process is hindered at low temperatures, and thus non-observable in that temperature range.

Since the fragility of a supercooled liquid is determined by the temperature dependence of the cooperative $\alpha$ relaxation and therefore also the liquid's viscosity, it is possible to use the fragility concept only when the $\alpha$ relaxation is visible. If the relaxation times of the QENS 
data would correspond to the $\alpha$ relaxation, a $\mathrm{T}_{g} \simeq 75 \mathrm{~K}$ would be obtained by extrapolation to $\tau \sim 100 \mathrm{~s}$. This value contradicts most previous work on supercooled water.

It is not possible to determine the cooperative $\alpha$ relaxation of supercooled water in small nanoporous silica and therefore the concept of fragility (including a FST) cannot be used. The abrupt change observed by QENS is thus not compatible with any of the proposed $\mathrm{T}_{g}$ 's. However a small change in the temperature dependence of the average relaxation time can be explained by the merging of a non-observable $\alpha$ relaxation and a local $\beta$ relaxation. Importantly, this does not rule out the possibility of a true FST of supercooled water in systems where the $\alpha$ relaxation is visible.

\section{References}

1. P.G. Debenedetti, J. Phys.: Condens. Matter 15, R1669 (2003)

2. C.A. Angell, E.J. Sare, J. Chem. Phys. 52, 1058 (1970)

3. G.P. Johari, A. Hallbrucker, E. Mayer, Nature (London) 330, 552 (1987)

4. S. Cerveny, G.A. Schwartz, R. Bergman, J. Swenson, Phys. Rev. Lett. 93, 245702 (2004)

5. V. Velikov, S. Borick, C.A. Angell, Science 294, 2335 (2001)

6. F.W. Starr, C.A. Angell, H.E. Stanley, Physica (Amsterdam) 323A, 51 (2003)

7. A. Minoguchi, R. Richert, C.A. Angell, Phys. Rev. Lett. 93, 215703 (2004)

8. K. Ito, C.T. Moynihan, C.A. Angell, Nature (London) 398, 492 (1999)

9. J. Swenson, R. Bergman, S. Longeville, J. Chem. Phys. 115, 11299 (2001)

10. H. Jansson, J. Swenson, Eur. Phys. J. E 12, S51 (2003)

11. A. Faraone, L. Liu, C.-Y. Mou, C.-W. Yen, S.-H. Chen, J. Chem. Phys. 121, 10843 (2004)

12. E. Mamontov, J. Chem. Phys. 123, 171101 (2005)

13. R. Bergman, J. Swenson, Nature (London) 403, 283 (2000)

14. R. Bergman, J. Swenson, L. Börjesson, P. Jacobsson, J. Chem. Phys. 113, 357 (2000)

15. J. Swenson, H. Jansson, W.S. Howells, S. Longeville, J. Chem. Phys. 122, 1 (2005)

16. L. Liu, S.-H. Chen, A. Faraone, C.-W. Yen, C.-Y. Mou, Phys. Rev. Lett. 95, 117802 (2005)

17. S. Takahara, M. Nakano, S. Kittaka, Y. Kuroda, T. Mori, H. Hamano, T. Yamaguchi, J. Phys. Chem. B 103, 5814 (1999)

18. T. Mori, Y. Kuroda, Y. Yoshikawa, M. Nagao, S. Kittaka, Langmuir 18, 1595 (2002)

19. A. Spanoudaki, B. Albela, L. Bonneviot, M. Peyrard, Eur. Phys. J. E 17, 21 (2005)

20. J. Hedström, J. Swenson, R. Bergman, S. Kittaka (to be published)

21. A. Arbe, A. Algeria, J. Colmenero, S. Hoffman, L. Willner, D. Richter, Macromolecules 32, 7572 (1999)

22. G. Williams, Adv. Polym. Sci. 33, 60 (1979)

23. R. Bergman, C. Svanberg, Phys. Rev. E 72, 043501 (2005) 